Año 11.

Frontera

Núm. 27
Revista de Investigación

Académica sin

ISSN: 2007-8870

http://revistainvestigacionacademicasinfrontera.com

Recibido el 15 de marzo de 2018. Dictaminado mediante arbitraje favorablemente 16 de mayo de 2018

\title{
Confiabilidad y validez de un instrumento que mide las corresponsabilidades hídricas
}

Reliability and validity of an instrument that measures water co-responsibilities

Oscar Valdés-Ambrosio ${ }^{1}$, José Marcos Bustos-Aguayo², Cruz García Lirios ${ }^{3}$

Resumen -La corresponsabilidad hídrica entendida como la culminación de un proceso de co-participación y consenso, así como el inicio de un proceso que prosigue con la cogestión y culmina con la coadministración, fue el objeto de investigación del presente trabajo. Se realizó un estudio no experimental con una selección no probabilística de 324 residentes de una localidad del centro de México en los que se observaron dos dimensiones que, sin embargo, el mismo diseño limitó los hallazgos al escenario de la investigación, sugiriendo la inclusión de un tercer factor relativo a la hipermetropía para explicar la despreocupación y la inacción ante los eventos de riesgo y las problemáticas hídricas locales.

Palabras clave -Escasez, desabastecimiento, recursos hídricos, servicios municipales, modelo

1 UNAM, Facultad de Economía: oscarva@unam.mx

2 UNAM, Facultad de Psicología: Marcos.bustos@unam.mx

3 UAEMEX, Facultad de Psicología: garcialirios@iloud.com 
Año 11.

Frontera

Núm. 27
Revista de Investigación

Académica sin

ISSN: 2007-8870

\title{
http://revistainvestigacionacademicasinfrontera.com
}

\begin{abstract}
Water co-responsibility, understood as the culmination of a process of coparticipation and consensus, as well as the beginning of a process that continues with comanagement and culminates with co-administration, was the object of research in this paper. A non-experimental study was carried out with a non-probabilistic selection of 324 residents of a locality in the center of Mexico where two dimensions were observed; however, the same design limited the findings to the research scenario, suggesting the inclusion of a third factor related to farsightedness to explain the lack of concern and inaction in the face of risk events and local water problems.
\end{abstract}

Keywords -Shortage, shortage, water resources, municipal services, model

\section{Introducción}

El objetivo del presente trabajo es establecer la confiabilidad y la validez de un instrumento que mide la corresponsabilidad hídrica en una localidad del centro de México, considerando su exposición a los eventos de riesgos como sequías, inundaciones y deslaves en relación con las problemáticas hídricas de escasez, desabastecimiento, insalubridad y carestía del servicio público o municipal.

La corresponsabilidad, para los fines del presente trabajo alude al establecimiento de una problemática común, una negociación entre las partes y un acuerdo entre los implicados con la finalidad de revertir la problemática o reducir su impacto en la salud pública, comunitaria o local, así como su reorientación al desarrollo endógeno o municipal (Corral, 2002a; 2002b; 2002c). 
Año 11.

Frontera

Núm. 27
Revista de Investigación

Académica sin

ISSN: 2007-8870

\section{http://revistainvestigacionacademicasinfrontera.com}

Sin embargo, la corresponsabilidad es un fenómeno observable en contextos de gobernanza y por consiguiente, sólo en demarcaciones muy específicas de civilidad en donde gobernantes y gobernados llevan a cabo negociaciones y cabildeos con la finalidad de anticipar conflictos, o bien, edificar un sistema de cogobierno (Corral, 2003a; 2003b; 2003c).

En tal contexto es que la gobernanza está en ciernes ya que, si bien en algunas colonias o municipios se ha incluido a la sociedad civil en temas como el de seguridad pública a través de las denuncias ciudadanas, la vigilancia civil y el seguimiento a los casos de reinserción social, en cuanto a la sustentabilidad los actores más bien han desarrollo una desconfianza mutua y han limitado el establecimiento de tarifas con base en la disponibilidad hídrica y no sólo en el volumen de consumo (García, 2005a; 2005b; 2005c; 2005d; 2005e; 2005f).

\section{Estudios de las corresponsabilidades hídricas}

Los estudios de la corresponsabilidad hídrica fueron en una escala global confinados a la calidad del servicio y en relación al sistema de cobro, así como al enjuiciamiento de actores políticos y civiles por ecocidio o daños al patrimonio natural y social. A medida que los desencuentros entre gobernantes y gobernados se exacerbaron surgieron propuestas de acuerdos con base en las tarifas diferencias acordes al consumo, las necesidades y las expectativas de los actores, pero sin dar solución a quienes se beneficiaron con el suministro sin haber pagado por el servicio, o bien, sin garantizar el uso sustentable y consumo de las futuras generaciones, legitimando la administración privada de los recursos hídricos (véase Tabla 1). 
(Enero- junio 2018)

"El saber de mis hijos

Revista de Investigación

Año 11.

Académica sin

Frontera

Núm. 27

ISSN: 2007-8870

\section{http://revistainvestigacionacademicasinfrontera.com}

Tabla 1. Estudios de la corresponsabilidad hídrica

\begin{tabular}{|c|c|c|}
\hline Añ & Autor & Hallazgo \\
\hline 2013 & García & $\begin{array}{l}\text { El sesgo de los medios impresos respecto a la inseguridad migratoria se caracterizó por un } \\
\text { encuadre significativamente menor en referencia al enmarcado en torno a otros ámbitos de } \\
\text { seguridad nacional y regional. Es decir, la difusión de la inseguridad migratoria en los } \\
\text { medios de comunicación parece corroborar el supuesto según el cual los medios impresos } \\
\text { construyen una agenda pública a partir de periodos electorales federales y comicios locales. } \\
\text { Realizó una búsqueda de fuentes periodísticas que cubrieron los conflictos relacionadas con } \\
\text { el servicio de agua potable en una demarcación de la Ciudad de México y encontró la } \\
\text { prevalencia de atribuciones a la mercadocracia. }\end{array}$ \\
\hline 2015 & $\begin{array}{l}\text { García, } \\
\text { Carreón y } \\
\text { Quintero }\end{array}$ & $\begin{array}{l}\text { Definieron la agenda institucional con base en criterios de administración consensuada de } \\
\text { los recursos y los servicios hídricos a fin de poder establecer los ejes de discusión centrados } \\
\text { en la baja disponibilidad y la creciente demanda de agua, el desabastecimiento y los } \\
\text { conflictos entre los actores políticos y sociales. }\end{array}$ \\
\hline 2015 & $\begin{array}{l}\text { Carreón et } \\
\text { al., }\end{array}$ & $\begin{array}{l}\text { Establecieron la asociación directa, positiva y significativa entre actitudes e intención de } \\
\text { voto a favor de propuestas de conservación de los recursos naturales en el marco de } \\
\text { elecciones federales presidenciales. }\end{array}$ \\
\hline 2017 & García et al., & $\begin{array}{l}\text { Encontraron diferencias significativas entre grupos estructurados según el sexo, edad, } \\
\text { ingreso, estado civil y tiempo de residencia con respecto al estrés y la resiliencia en un } \\
\text { contexto de inundaciones por el desborde de un rio aledaño a la comunidad de estudio, } \\
\text { catalogada como vulnerable a los efectos del cambio climático sobre la salud pública } \\
\text { ambiental. }\end{array}$ \\
\hline 2017 & $\begin{array}{l}\text { Sandoval et } \\
\text { al., }\end{array}$ & $\begin{array}{l}\text { Establecieron la confiabilidad y la validez de un instrumento que mide la percepción de } \\
\text { riesgos, estrés y resiliencia, encontrando que la edad determinó la percepción de riesgos y } \\
\text { ésta la resiliencia. Es decir que los residentes adultos mayores desarrollan una mayor } \\
\text { percepción de riesgo que les anticipa a afrontar las contingencias del entorno en la medida } \\
\text { en que se exacerban lluvias, inundaciones o deslaves. }\end{array}$ \\
\hline 2017 & Carreón & $\begin{array}{l}\text { Estableció una secuencia de minería de datos con las notas de prensa de circulación nacional } \\
\text { relativas a la seguridad pública, encontrando el establecimiento de verosimilitud como } \\
\text { lógica de persuasión de los medios. }\end{array}$ \\
\hline 2017 & García & $\begin{array}{l}\text { Revisó y analizó la literatura concerniente al establecimiento de la agenda como tema } \\
\text { central del estado del conocimiento en el que el encuadre ocupó el segundo sitio y los }\end{array}$ \\
\hline
\end{tabular}




\section{http://revistainvestigacionacademicasinfrontera.com}

\begin{tabular}{|c|c|c|}
\hline 2018 & $\begin{array}{l}\text { Sandoval, } \\
\text { Bustos y } \\
\text { García }\end{array}$ & $\begin{array}{l}\text { Establecieron un modelo factorial exploratorio en el que las intenciones de llevar a cabo un } \\
\text { acto político relacionado con los servicios hídricos y la optimización del abastecimiento de } \\
\text { agua potable fue determinado por las disposiciones a favor del sistema electoral y las } \\
\text { creencias de afectación de los comicios. }\end{array}$ \\
\hline 2018 & $\begin{array}{l}\text { García, } \\
\text { Juárez y } \\
\text { Bustos }\end{array}$ & $\begin{array}{l}\text { Especificaron un modelo para el estudio de la gobernanza de la sustentabilidad local a partir } \\
\text { del emprendimiento y la innovación social, así como en relación a la seguridad, principales } \\
\text { factores del desarrollo local. }\end{array}$ \\
\hline 2018 & $\begin{array}{l}\text { Quintero et } \\
\text { al., }\end{array}$ & $\begin{array}{l}\text { Establecieron cinco factores relativos a la conciencia para la gobernanza de la } \\
\text { sustentabilidad, habilidades, conocimientos, motivos y disposiciones de corresponsabilidad. } \\
\text { Se trata de un modelo centrado en la cognición social como factor preponderante en la } \\
\text { negociación, acuerdos y responsabilidades compartidas. }\end{array}$ \\
\hline 2018 & $\begin{array}{l}\text { Vanegas et } \\
\text { al., }\end{array}$ & $\begin{array}{l}\text { Demostró la incidencia de las expectativas de comportamiento proambiental sobre el } \\
\text { comportamiento proambiental, así como una correlación negativa con respecto a las } \\
\text { expectativas de comportamiento anti-ambiental. }\end{array}$ \\
\hline $\begin{array}{l}2012 a ; \\
2012 b\end{array}$ & García & $\begin{array}{l}\text { Demostró el efecto agenda en los medios impresos locales con respecto a denuncias y } \\
\text { conflictos en una demarcación con baja disponibilidad hídrica y alto índice de comercio } \\
\text { informal de agua. La percepción mediática del accionar gubernamental incidió } \\
\text { positivamente sobre la percepción de la inseguridad pública }(\beta=.36 ; \mathrm{p}<.001)\end{array}$ \\
\hline $\begin{array}{r}2014 a ; \\
2014 b\end{array}$ & $\begin{array}{l}\text { Carreón et } \\
\text { al., }\end{array}$ & $\begin{array}{l}\text { Demostraron que los conflictos hídricos están centrados en las condonaciones de pago y la } \\
\text { proximidad de los comicios locales como federales en México. Establecieron criterios de } \\
\text { análisis de contenido de notas de prensa respecto a los conflictos hídricos vinculados a las } \\
\text { elecciones locales y el apoyo a partidos como a candidatos ecologistas. Especificaron un } \\
\text { modelo de agenda sociopolítica para el estudio de la gobernanza de los recursos y los } \\
\text { servicios hídricos, así como la medición del consumo y el cobro con aumento de tarifas. } \\
\text { Establecieron criterios de análisis de contenido de notas de prensa respecto a los conflictos } \\
\text { hídricos vinculados a las elecciones locales y el apoyo a partidos como a candidatos } \\
\text { ecologistas. La TRS advertiría que los recursos al ser transformados en servicios públicos } \\
\text { no sólo son exclusivos de la especie humana, sino sólo de quienes pueden costear su valor } \\
\text { de uso y valor de cambio }\end{array}$ \\
\hline $\begin{array}{l}2015 a ; \\
2015 b ; \\
2015 c\end{array}$ & García et al., & $\begin{array}{l}\text { Establecieron diferencias significativas entre caficultores con respecto a sus expectativas de } \\
\text { riesgo ante los efectos del cambio climático en la seguridad alimenticia, principalmente la } \\
\text { agricultora local como es el caso de inundaciones, sequías o deslaves que afectaron la }\end{array}$ \\
\hline
\end{tabular}




\section{http://revistainvestigacionacademicasinfrontera.com}

producción agrícola y el comercio local como regional. Se revisó el estado de la cuestión en torno al establecimiento de una agenda local en materia de sustentabilidad hídrica, centrada en las tarifas y la calidad del servicio, concluyendo que la gestión ha sido rebasada por la demanda y la acción colectiva municipal. Vincularon el establecimiento de agenda con la gobernanza de los servicios hídricos en una situación de escasez y desabastecimiento de agua reportada en la prensa de circulación nacional. Demostraron la tendencia de una propaganda a favor de la administración pública del agua, pero una gestión privada en cuanto a yacimientos, bombeo y distribución.

2016a; García et al., Encontraron dos factores relativos a la calidad del servicio de abastecimiento de agua

$2016 b$;

2016c;

2016d potable y el sistema de tarifas, subsidios y condonaciones en el marco de futuras elecciones, los resultados anticipan un escenario de contienda electoral centrada en la promesa de abastecimiento regular y disminución de las tarifas, aumento de subsidios y condonaciones a cambio del apoyo electoral del partido o candidato a gobernar la demarcación. Las representaciones sociales del tandeo consisten en emplazamientos para la acción colectiva, la movilización social y la participación comunitaria en torno al abastecimiento de agua, el consumo y pago del servicio. Revisaron la literatura concerniente a la gestión y la administración de los recursos y los servicios hídricos municipales, estableciendo ejes de discusión y escenarios de análisis sociopolítico en torno a la corresponsabilidad en la preservación del agua, pero evidenciando las asimetrías entre los actores políticos y sociales con respecto a la responsabilidad social y el establecimiento de una agenda pública. Se establecieron premisas relativas al establecimiento de una agenda local con respecto a las representaciones sociales de residentes periurbanos en torno al sistema de cobro y abastecimiento público de agua potable, encontrándose una prevalencia de los conflictos entre los actores políticos y civiles.

2018a; García

$2018 b$;

$2018 c$
Especificó un modelo con fuentes de 1972 a 2017, indexadas a repositorios líderes de América Latina, estableciendo cinco factores: corresponsabilidad hídrica, agenda sociopolítica, propaganda de bienes comunes, emergencia del tequio y la guatza, así como la cognición social hídrica. Interpretó los discursos de usuarios con la finalidad de comprender sus narrativas alusivas a la gratuidad de acceso y uso del agua como servicio público y la obligación del Estado como eje rector de la gestión y la administración de los recursos hídricos, encontrando la legitimidad de las políticas de oferta frente a las iniciativas civiles de austeridad y los estilos de frugalidad locales.

Fuente: Elaboración propia 
Año 11.

Frontera

Núm. 27
Revista de Investigación

Académica sin

ISSN: 2007-8870

\section{http://revistainvestigacionacademicasinfrontera.com}

Pues bien, en la medida en que la sociedad civil renunció a su estilo de vida de confort y recreación hídrica fue tomando más conciencia de las problemáticas. En tal sentido es que las propuestas más formales de negociación, acuerdos, conciliación, arbitraje y enjuiciamiento proliferaron (García, 2006a; 2006b).

Pues bien, los estudios de la corresponsabilidad avanzaron hacia la edificación de una agenda centrada en la deliberación e inversión pública, así como en la transparencia de la cuestión y la impartición de justicia a gobernantes y gobernados por hidro-corrupción como es el caso de la sobreexplotación de los mantos acuíferos, la contaminación de cuencas o la discrecionalidad en la designación de partidas a hidroeléctricas (García, 2007a; 2007b).

Los estudios de la corresponsabilidad hídrica avanzaron hacia el establecimiento de una agenda común entre autoridades y usuarios del sistema, sectores públicos y privados en la medida en que se vinculó el establecimiento de temas a la proximidad electoral (García, 2009a; 2009b; 2009c).

En tal coyuntura, los actores sociales y políticos desarrollaron una agenda común pero asimétrica para los sectores excluidos de las organizaciones civiles y políticas que no tuvieron voz ni voto en los proyectos hídricos ni presionaron mediante el voto en las elecciones (García, 2010a; 2010b).

Sin embargo, la emergencia de las redes sociales, así como de los dispositivos electrónicos abrió la posibilidad de manifestar y orientar las demandas hacia espacios de discusión y difusión del sentir político y social que penetraron las instituciones encargadas de la sustentabilidad hídrica (García, 2011a; 2011b; 2011c; 2011d; 2011e; 2011f). 
Año 11.

Frontera

Núm. 27
Revista de Investigación

Académica sin

ISSN: 2007-8870

\section{http://revistainvestigacionacademicasinfrontera.com}

En esa medida, la corresponsabilidad se gestó en un clima electoral, pero se consolidó en los medios de comunicación tradicionales en la medida en que las encuestadoras registraron tendencias e intenciones de voto en las redes sociales y digitales (García, 2012a; 2012b).

Es así como la corresponsabilidad hídrica está asociada a los medios de comunicación en general y las redes electrónicas en particular, esencialmente en contiendas electorales donde prevalecen rezagos en materia de gobernanza de la sustentabilidad hídrica (Bustos, Juárez, Sandoval, Quintero, y García, 2017).

Por consiguiente, la corresponsabilidad hídrica está dimensionada en una aversión a la gestión estatal y la autogestión civil, así como en una propensión a la coparticipación, la cogestión y el consenso, pero no sólo observable en una asamblea o mitin, sino además verificable en las publicaciones de Facebook, Twitter, Instagram, WhatsApp, SnapChat, YouTube o Periscope (Carreón, Hernández, Morales, Rivera, y García, 2014).

A pesar de tal evidencia, los estudios de la corresponsabilidad hídrica no se han desarrollado en ese ámbito y sólo han abordado los sondeos u opiniones a usuarios ante fugas públicas, conflictos con las autoridades como el cierre de avenidas, el secuestro de pipas o la venta de agua (García, Rivera, Limón, Bustos, y Juárez, 2017).

\section{Método}

¿Existen diferencias significativas entre las dimensiones teóricas de la corresponsabilidad hídrica con respecto a las relaciones entre sus factores e indicadores a ser observados en un estudio empírico? 
Año 11.

Frontera

Núm. 27
Revista de Investigación

Académica sin

ISSN: 2007-8870

\section{http://revistainvestigacionacademicasinfrontera.com}

Hipótesis nula: Existirán diferencias significativas entre los factores teóricos con respecto a las dimensiones empíricas a ser observadas en el estudio

Hipótesis alterna: No existirán diferencias significativas entre las dimensiones teóricas de la corresponsabilidad hídrica en relación con sus dimensiones empíricas a ser observadas en un estudio local.

Se realizó un estudio no experimental con una selección no probabilística de 324 residentes de una colonia del oriente de la Ciudad de México, considerando su bajo nivel de desarrollo humano y alta prevalencia de escasez, desabastecimiento, insalubridad y carestía del servicio público (véase Tabla 2).

Tabla 2. Descriptivos de la muestra

\begin{tabular}{|llllllll|} 
Sexo & $\%$ & Edad & $\%$ & ingreso & $\%$ & Estado Civil & $\%$ \\
\hline Masculino & 54,2 & $>22$ años & 46,2 & $>300$ USD & 52,1 & Soltería & 67,2 \\
\hline Femenino & 56,8 & $<22$ años & 64,8 & $<300$ USD & 58,9 & Otro & 43,8
\end{tabular}

Fuente: Elaborada con los datos del estudio

Se construyó la Escala de Corresponsabilidad Hídrica (ECH-14) la cual incluyó las dos dimensiones con cinco opciones de respuesta que van desde $0=$ "nada probable" hasta $5=$ "bastante probable" (véase Tabla 3). 
Año 11.

Frontera

Núm. 27
Académica sin

ISSN: 2007-8870

\section{http://revistainvestigacionacademicasinfrontera.com}

Tabla 3. Construcción de la ECH-14

\begin{tabular}{|c|c|c|c|c|}
\hline Pactor & Concepto & Indicador & Medición & Interpretación \\
\hline Aversión & $\begin{array}{l}\text { Refiere a las expectativas a } \\
\text { favor de la autogestión civil } \\
\text { y la gestión estatal (García, } \\
\text { Carreón y Hernández, } \\
\text { 2017). }\end{array}$ & $\begin{array}{l}\text { Asumiré un rol más } \\
\text { pasivo ante una } \\
\text { inflación de las tarifas }\end{array}$ & $\begin{array}{l}0 \quad=\text { "nada } \\
\text { probable", } \\
\text { hasta } 5= \\
\text { "bastante } \\
\text { probable", }\end{array}$ & $\begin{array}{l}\text { Un alto puntaje } \\
\text { refiere a una barrera } \\
\text { para la gobernanza }\end{array}$ \\
\hline Propensión & $\begin{array}{l}\text { Refiere a las expectativas } \\
\text { favorables a la } \\
\text { coparticipación y la } \\
\text { cogestión (García, Carreón, } \\
\text { Bustos y Juárez, 2016). }\end{array}$ & $\begin{array}{l}\text { Demandaré a mis } \\
\text { representantes una } \\
\text { tarifa universal del } \\
\text { servicio municipal }\end{array}$ & $\begin{array}{l}0 \quad=\text { "nada } \\
\text { probable", } \\
\text { hasta } 5= \\
\text { "bastante } \\
\text { probable", }\end{array}$ & $\begin{array}{l}\text { Un alto puntaje alude } \\
\text { a una orientación } \\
\text { hacia la gobernanza }\end{array}$ \\
\hline
\end{tabular}

Fuente: Elaboración propia

Se encuestaron a los participantes en las instalaciones de la universidad pública del centro de México, previa garantía de confidencialidad, anonimato y no afectación a su estatus académico. La información se procesó en el Paquete Estadístico para Ciencias Sociales (IBM-SPSS-AMOS versión 25).

Se realizaron análisis multivariables de normalidad, confiabilidad y validez, así como la adecuación y esfericidad para el ajuste y residual de la prueba de hipótesis nula. 
Año 11.

Frontera

Núm. 27
Revista de Investigación

Académica sin

ISSN: 2007-8870

\section{http://revistainvestigacionacademicasinfrontera.com}

\section{Resultados}

La Tabla 4 muestra los valores de consistencia interna de la ECH-14 (alfa de ,780) y las subescalas de los factores de aversión (alfa de ,785) y propensión (alfa de ,788), así como los pesos factoriales de los indicadores con respecto a las dimensiones de la corresponsabilidad hídrica.

Tabla 4. Descriptivos del ECH-14

\begin{tabular}{llllllll}
\hline $\mathbf{R}$ & M & D & S & C & A & F1 & F2 \\
\hline R1 & 4,38 & 1,45 & 1,43 & 1,49 &, 782 & &, 418 \\
\hline R2 & 4,30 & 1,31 & 1,32 & 1,34 &, 740 &, 438 \\
\hline R3 & 4,32 & 1,24 & 1,46 & 1,02 &, 761 &, 438 \\
\hline R4 & 4,56 & 1,36 & 1,49 & 1,06 &, 780 &, 438 \\
\hline R5 & 4,76 & 1,45 & 1,40 & 1,02 &, 783 & &, 415 \\
\hline R6 & 4,95 & 1,35 & 1,83 & 1,04 &, 762 & &, 403 \\
\hline R7 & 4,56 & 1,12 & 1,78 & 1,01 &, 740 & &, 439 \\
\hline R8 & 4,35 & 1,47 & 1,26 & 1,02 &, 761 &, 436 & \\
\hline R9 & 4,41 & 1,29 & 1,40 & 1,04 &, 748 &, 401 & \\
\hline R10 & 4,30 & 1,30 & 1,56 & 1,08 &, 752 &, 436 & \\
\hline R11 & 4,56 & 1,31 & 1,81 & 1,05 &, 747 &, 467 & \\
\hline R12 & 4,38 & 1,38 & 1,40 & 1,06 &, 731 &, 491 & \\
\hline R13 & 4,60 & 1,24 & 1,24 & 1,07 &, 736 &, 436 & \\
\hline R14 & 4,36 & 1,29 & 1,41 & 1,08 &, 751 &, 462 & \\
\hline
\end{tabular}


Año 11.

Frontera

Núm. 27
Revista de Investigación

Académica sin

ISSN: 2007-8870

\section{http://revistainvestigacionacademicasinfrontera.com}

$\mathrm{R}=$ Reactivo, $\mathrm{M}=$ Media, $\mathrm{D}=$ Desviación, $\mathrm{S}=$ Sesgo, $\mathrm{C}=$ Curtosis, $\mathrm{A}=$ Alfa de Crombach quitando el valor del ítem, Adecuación $(\mathrm{KMO}=, 752)$, Esfericidad $\int \mathrm{X}^{2}=345,23(56 \mathrm{gl}) \mathrm{p}=$ ,000 J . Extracción: Ejes Principales, Rotación: Promax. F1 = Aversión a la Gobernanza de la Sustentabilidad Hídrica $(25 \%$ de la varianza total explicada), F2 = Propensión a la Gobernanza de la Sustentabilidad Hídrica (16\% de la varianza total explicada). Todos los ítems se responden con una de cinco opciones: $0=$ "nada probable" hasta $5=$ "bastante probable".

Fuente: Elaborada con los datos del estudio

Los parámetros de adecuación $(\mathrm{KMO}=, 752)$ y la prueba de esfericidad $\int \mathrm{X}^{2}=345,23$ $(56 \mathrm{gl}) \mathrm{p}=, 000 \mathrm{~J}$ sugierieron un análisis factorial exploratorio de ejes principales con una rotación promax.

Una vez establecidos los dos factores que explicaron el $41 \%$ de la varianza total se contrastó la hipótesis nula. Los parámetros de ajuste y residual $\int \mathrm{X}^{2}=453,24$ (49 gl) $\mathrm{p}=$ ,007; GFI = ,997; CFI = ,990; RMSEA = ,008 J sugierieron el no rechazo de la hipótesis según la cual existirán diferencias significativas entre las dimensiones teóricas de la corresponsabilidad con respecto a las relaciones entre lso factores y los indicadores observados en la investigación empírica.

\section{Discusión}

El aporte del presente trabajo al estado de la cuestión radica en el establecimiento de la confiabilidad y la validez de un instrumento que mide la corresponsabilidad hídrica en sus dos dimensiones de aversión y propensión a la gobernanza, aunque el tipo de estudio no experimental, el tipo de muestreo no probabilístico y el tipo de análisis no estructural 
Año 11.

Frontera

Núm. 27
Revista de Investigación

Académica sin

ISSN: 2007-8870

\section{http://revistainvestigacionacademicasinfrontera.com}

limitaron los resultados al escenario de la investigación, sugiriendo la inclusión de un tercer factor que la literatura identifica como hipermetropía para explicar la despreocupación y la inacción ante eventos de riesgo y problemáticas ambientales.

García (2018a; 2018b; 2018c) advierte que la gobernanza de la sustentabilidad hídrica local depende de la negociación y el consenso con respecto a las problemáticas hídricas de escasez, desabastecimiento, insalubridad y carestía, pero en esta investigación se ha demostrado que la gobernanza depende de la corresponsabilidad en su dimensión de aversión y propensión a la gestión estatal como a la autogestión civil.

Vanegas, Corral Bustos y Ortega (2018) a través de un modelo estructural $\left\lceil X^{2}=136,34\right.$ $(86$ gl $) \mathrm{p}<, 001 ; \mathrm{CFI}=.995 ; \mathrm{NNFI}=, 993 ; \mathrm{RMSEA}=, 005 ; \mathrm{R}^{2}=, 04 \mathrm{~J}$ el efecto de las expectativas de conducta proambiental con respecto al comportamiento proambiental $(, 28)$ y una asociación con las expectativas de conducta antiambiental $(-, 86)$ que para el presente estudio son relevantes ya que la aversión y la propensión a la gobernanza parece depender de una expectativa de comportamiento proambiental, aunque las expectativas de conducta antiambiental supondrían más bien una primera fase del establecimiento de la agenda para la edificación de la gobernanza de la sustentabilidad hídrica.

Sandoval, Bustos y García (2018) mediante una estructura factorial exploratoria $\lceil\mathrm{X} 2=$ 432,13 (35 gl) p =,021; GFI = ,995; CFI = ,990; RMSEA = ,007; $\eta^{2}=, 21 \mathrm{~J}$ establecieron la incidencia de los valores biosféricos sobre las intenciones de consenso que, para el presente trabajo es fundamental ya que la aversión como la propensión de la gobernanza de la sustentabilidad hídrica supone valores biosféricos, egoístas y altruistas que converjan en una conciencia de los bienes comunes para la edificación de un futuro sostenible. 
Año 11.

Frontera

Núm. 27
Revista de Investigación

Académica sin

ISSN: 2007-8870

\section{http://revistainvestigacionacademicasinfrontera.com}

Es menester introducir las variables que la literatura advierte como colaterales al proceso deliberado, planificado y sistemático de gobernanza de la sustentabilidad hídrica local, así como el contraste del modelo en otros escenarios y muestras.

\section{Conclusión}

El objetivo del presente trabajo ha sido establecer la confiabilidad y la validez de un instrumento que mide la corresponsabilidad como factor de la gobernanza de la sustentabilidad hídrica local, aunque el tipo de estudio, muestreo y análisis limitan los resultados al escenario de la investigación, así como la inclusión de otros factores que la literatura identifica como hipermetropía o despreocupación e inacción ante los eventos de riesgo y los problemas hídricos locales.

\section{Referencias}

Bustos, J. (2004). Modelo de conducta proambiental para el estudio de la conservación de agua potable. Tesis de Doctorado, Facultad de Psicología, Universidad Nacional Autónoma de México.

Bustos, J. Flores, M. y Andrade, P. (2004). Predicción de la conservación de agua a partir de factores socio cognitivos. Medio Ambiente y Comportamiento Humano. 5, 53-70

Bustos, J. M., Juárez, M., Sandoval, F. R., Quintero, M. L. y García, C. (2017). Percepciones sobre la calidad y las tarifas del servicio de agua potable de los usuarios de la ciduad de México. Revista Educación y Desarrollo Social, 11 (2), 20-31

Bustos, J. y Flores, L. (2000). Evaluación de problemas ambientales, calidad del ambiente y creencias de afectación de la salud. La Psicología Social en México. 8, 445-451. 
Año 11.

Frontera

Núm. 27
Revista de Investigación

Académica sin

ISSN: 2007-8870

\section{http://revistainvestigacionacademicasinfrontera.com}

Bustos, J., Flores, L. y Andrade, P. (2002). Motivos y percepción de riesgos como factores antecedentes a la conservación de agua en la Ciudad de México. La Psicología Social en México. 9, 611-617.

Bustos, J., Flores, L., Barrientos, C. y Martínez, J. (2004). Ayudando a contrarrestar el deterioro ecológico: atribución y motivos para conservar agua. La Psicología Social en México. 10, 521-526

Bustos, J., Montero, M. y Flores, L. (2002). Tres diseños de intervención antecedente para promover conducta protectora del ambiente. Medio Ambiente y Comportamiento Humano. 3, 63-88.

Carreón, J., Bustos, J. M., García, C., Hernández, J. y Mendoza, D. (2015). Utilización de SPSS y AMOS en un estudio del pensamiento ambientalista y las intenciones de voto en una muestra de estudiantes. Multidisciplina, 20, 76-95

Carreón, J., García, C., Morales, M. L. (2014). Hacia una administración consensuada de los recursos hídricos en ecociudades. Interdisciplinaria, 31 (1), 163-174

Carreón, J., García, C., Morales, M. L. y Domínguez, G. A. (2011). Teorías psicosociales para explicar los conflictos derivados del abastecimiento de agua en México, Distrito Federal. Pampedia, 8, 56-68

Carreón, J., Hernández, J. y García, C. (2014). Análisis de notas de prensa respecto a condonaciones por pago de servicios hídricos. Doxa, 47 (8), 134-152 
Año 11.

Frontera

Núm. 27
Revista de Investigación

Académica sin

ISSN: 2007-8870

\section{http://revistainvestigacionacademicasinfrontera.com}

Carreón, J., Hernández, J., García, C., Bustos, J. M., Morales, M. 1. y Aguilar, J. A. (2014).

La psicología de la sustentabilidad hídrica: Políticas públicas y modelos de consumo. Aposta, 63, 1-29

Carreón, J., Hernández, J., García, C., Rivera, B. L. y Morales, M. L. (2014). Análisis de notas de prensa en torno al encuadre sociopolítico de tarifas hídricas. Obets, 9 (1), 73 94

Carreón, J., Hernández, J., Morales, M. L., Rivera, B. 1. y García, C. (2014). Intenciones de voto a favor de propuestas verdes ¿Nuevo paradigma ambiental y posmaterialismo social? Revista de Psicología Política, 12 (32), 37-54

Corral, V \& Frías, M. (2006). Personal normative beliefs, antisocial behavior, and residential water conservation. Environment and Behavior. 38, 406-421

Corral, V. (1997). Un análisis crítico del concepto "actitudes" parte 1: postulados y métodos de estudio. Revista Mexicana de Análisis de la Conducta. 23, 215-235.

Corral, V. (1998). Interacciones ambientes / conducta: algunas áreas de investigación. En V. M. Alcaraz y A. Bouzas (coord.). Las aportaciones mexicanas a la psicología. (pp. 55-70) México: UNAM.

Corral, V. (2000). La definición del Comportamiento Proambiental. La Psicología Social en México. 8, 466-472.

Corral, V. (2001). Aplicaciones del Modelamiento Estructural a la investigación psicológica. Revista Mexicana de Psicología. 18 193-209. 
Año 11.

Frontera

Núm. 27
Revista de Investigación

Académica sin

ISSN: 2007-8870

\section{http://revistainvestigacionacademicasinfrontera.com}

Corral, V. (2002a). A structural model of proenvironmental competency. Environment \& Behavior. 34, 531-549.

Corral, V. (2002b). Avances y limitaciones en la medición del comportamiento proambiental. en J. Guevara, y S. Mercado, (coords.) Temas Selectos de Psicología Ambiental. (pp. 483-510). México: UNAM-GRECO-UNILIBRE.

Corral, V. (2002c). Structural Equation Modelling. in Bechtel, R y Churcman, A. (eds.) Handbook of Environmental Psychology. (pp. 256-270). New York: Wiley \& Sons, Inc.

Corral, V. (2003a). ¿Mapas cognitivos o conductas ambientales? en E. Díaz, y M. Anaya, (coord.). Perspectivas sobre el cognitivismo en psicología. (pp. 37-79). México: UNAM (Iztacala).

Corral, V. (2003b). Determinantes psicológicos o situacionais do comportamento de conservaçào de àgua: um modelo estructural. Estudos de Psicología. 8, 245-252.

Corral, V. (2003c). Situational and personal determinants of waste control practices in Northern Mexico: a study of reuse and recycling behaviors. Recourses, Conservation \& Recycling. 39,265-281.

Corral, V. y Encinas, L. (2001). Variables disposicionales, situacionales y demográficas en el reciclaje de metal y papel. Medio Ambiente y Comportamiento Humano. 2, 1-19.

Corral, V. y Obregón, F. J. (1992). Modelos predictores del comportamiento proambientalista. Revista Sonorense de Psicología. 6, 5-14. 
Año 11.

Frontera

Núm. 27
Revista de Investigación

Académica sin

ISSN: 2007-8870

\section{http://revistainvestigacionacademicasinfrontera.com}

Corral, V. y Pinheiro, J. (1999). Condicoes para o estudo do comportamento próambiental. Estudos de Psicología. 4, 7-22.

Corral, V. y Pinheiro, J. (2004). Aproximaciones al estudio de la conducta sustentable. Medio Ambiente y Comportamiento Humano. 5, 1-26.

Corral, V. y Zaragoza, F. (2000) Bases sociodemográficas y psicológicas de la conducta de reutilización: Un Modelo estructural. Medio Ambiente y Comportamiento Humano. 1, 9-29.

Corral, V., Bechtel, R. \& Fraijo, B. (2003). Environmental beliefs and water conservation: an empirical study. Journal of Environmental Psychology. 23, 247-257.

Corral, V., Bechtel, R., Armendáriz, L. I. y Esquer, A. B. (1997). La estructura de las creencias ambientales en universitarios mexicanos: el Nuevo Paradigma Ambiental. Revista Mexicana de Psicología. 14, 173-181.

Corral, V., Capdevielle, F., Garibaldi, L. y Encinas, 1. (1986). Estrategias conceptuales para la reducción de consumo doméstico de agua en una zona urbana. La Psicología Social en México. 1, 475-479.

Corral, V., Fraijó, B. Y Tapía, C. (2004). Propensiones psicológicas en niños de sexto grado de primaria. Validez de un instrumento. Anuario de Investigaciones Educativas. $7,31-44$

Corral, V., Fraijo, B., Frías, M., González, D. y Pinheiro, J. (2004). Propensión al presente, al pasado y al futuro y sus relaciones con el ahorro de agua. La Psicología Social en México. 10, 547-552 
Año 11.

Frontera

Núm. 27
Revista de Investigación

Académica sin

ISSN: 2007-8870

\section{http://revistainvestigacionacademicasinfrontera.com}

Corral, V., Frías, M. \& González, D. (2001). On the relationship between antisocial and anti-environmental behaviors: an empirical study. Population and Environment. 24, 273-286.

Corral, V., Frías, M. y González, D. (2003). Percepción de riesgos, conducta proambiental y variables demográficas en una comunidad de Sonora, México. Región y Sociedad. $26,49-72$

Corral, V., Garibaldi, L. y Encinas, L. (1987). Estudio exploratorio de patrones conductuales de consumo doméstico de agua en zona urbana. Revista Sonorense de Psicología. 2, 87-93.

Corral, V., Varela, C. y González, D. (2002). Una taxonomía funcional de competencias proambientales. La Psicología Social en México. 9, 592-597.

García, C. (2004). La psicología ambiental del agua en el año 2025. Bricolage, 6, 19-27

García, C. (2005a). ¿Cuáles son los determinantes psicológicos y situacionales de un uso sustentable del agua en la Zona Metropolitana del Valle de México? Revista de Investigación y Educación, 15, 1-44

García, C. (2005b). ¿Qué sentimientos activan el recuerdo de abastecimiento y el cuidado de agua más como un elemento comunitario que como un recurso natural? Psicología, $9(1), 1-22$

García, C. (2005c). Dos modelos para explicar el uso sustentable del agua en la zona metropolitana del valle 
Año 11.

Frontera

Núm. 27
Revista de Investigación

Académica sin

ISSN: 2007-8870

\section{http://revistainvestigacionacademicasinfrontera.com}

ios psicológicos de la sustentabilidad. Nneme, 6 (13), 1-63

García, C. (2005e). Un modede México. Revista de Psicología Iztacala, 8 (1), 1-39

García, C. (2005d). Los estudlo para explicar el uso de agua en la Zona Metropolitana del Valle de México. Revista Internacional de Psicología, 6 (2), 1-138

García, C. (2005f). Un modelo psicosocial para promover el ahorro de agua en la zona metropolitana del valle de México. Ciencias Sociales, 11 (2), 103-109

García, C. (2006a). Un programa de uso sustentable del agua. Primera parte. Residuos, 90, $1-14$

García, C. (2006b). Un programa de uso sustentable del agua. Segunda parte. Residuos, 92, $1-14$

García, C. (2007a). Aproximaciones al uso sustentable del agua. Aqua Forum, 11 (44), 1620

García, C. (2007b). Un programa de conservación de agua. Entelequía, 5, 169-196

García, C. (2009a). Las investigaciones causales de las ciencias sociales en torno a las problemáticas hídricas. Gaceta de Antropología, 25 (2), 1-9

García, C. (2009b). Las investigaciones correlacionales de las ciencias sociales en torno a las problemáticas hidrológicas. Barataria, 10, 141-147 
Año 11.

Frontera

Núm. 27
Revista de Investigación

Académica sin

ISSN: 2007-8870

\section{http://revistainvestigacionacademicasinfrontera.com}

García, C. (2009c). Las teorizaciones de las ciencias sociales en torno a las problemáticas hidrológicas. Entelequia, 9, 63-98

García, C. (2010a) la creencia hidrológica en Iztapalapa. Revista de Psicología Política, 8 (24), 18-34

García, C. (2010b). La exclusión hidrológica. Entelequia, 11, 41-59

García, C. (2011a). La estructura del antropocentrismo hídrico. Multidisciplina, 10, 33-42

García, C. (2011b). La preocupación hidrológica en Iztapalapa. Revista de Psicología Gерри, 2 (1), 52-63

García, C. (2011c). La valoración hidrológica en Iztapalapa. Epsys, 3, 1-9

García, C. (2011d). Las estructuraciones de la sustentabilidad hídrica. Tlatemoani, 7, 1-27

García, C. (2011e). Las investigaciones comparativas de las ciencias sociales en torno a las problemáticas hídricas. Revista de Desarrollo local Sostenible, 4 (11), 1-12

García, C. (2011f). Las investigaciones estructurales en torno a las problemáticas hídricas. Revista de Psicología Gepu, 2 (2), 99-112

García, C. (2012a). La cobertura de la prensa en torno a denuncias, abastecimientos y emplazamientos ante una escasez de agua en Iztapalapa, México. Sociedad Hoy, 22, 995-113

García, C. (2012b). Los constructos de la sustentabilidad hídrica. Epsys, 3, 1-19 
Año 11.

Frontera

Núm. 27
Revista de Investigación

Académica sin

ISSN: 2007-8870

\section{http://revistainvestigacionacademicasinfrontera.com}

García, C. (2013). El encuadre periodístico de en torno a los conflictos hídricos derivados de la mercadocracia en México. Psicología para América Latina, 24, 121-155

García, C. (2018a). Especificación de un modelo con fuentes de 1987 a 2017 para el estudio de la corresponsabilidad hídrica en una localidad del centro e México. Diálogos de Derecho y Política, 19, 18-38

García, C. (2018b). Interpretación e discursos en torno al subsidio del servicio de abastecimiento hídrico para la comprensión de narrativas tarifarias. Ciencias Sociales, $4(2), 25-40$

García, C. (2018c). Teoría del comportamiento sustentable para el desarrollo local. Clivajes, 5 (9), 71-94

García, C. y Bustos, J. M. (2013). Los estudios psicológicos de la sustentabilidad hídrica. Aplicaciones al sistema tarifario de consumo. Revista de Ciencias Sociales, 139, 6590

García, C., Aguilar, J. A., Rosas, F. J., Carreón, J. y Hernández, J. (2015). Diferencias de fiabilidad sociopolítica ante conflictos hídricos entre actores civiles. Invurnus, 10 (2), $3-13$

García, C., Bustos, J. M., Juárez, M. Rivera, B. L. y Limón, G. A. (2017). Expectativas de usuarios del servicio de agua potable en torno al abastecimiento, la calidad y las tarifas en el marco de futuras elecciones en una localidad de la Ciudad de México. Compendium, 4 (7), 45-54 
Año 11.

Frontera

Núm. 27
Revista de Investigación

Académica sin

ISSN: 2007-8870

\section{http://revistainvestigacionacademicasinfrontera.com}

García, C., Carreón, J. y Hernández, J. (2017). La cogestión como dispositivo de seguridad para el desarrollo sustentable local. Eureka, 14 (2), 268-289

García, C., Carreón, J. y Quintero, M. L. (2015). Dimensiones de gobernanza de la sustentabilidad hídrica. Pueblos y Fronteras, 10 (20), 195-203

García, C., Carreón, J., Bustos, J. M. y Juárez, M. (2016). Escenarios relativos al establecimiento de agenda para la gobernanza transgeneracional de los recursos y servicios hídricos. Civilizar, 16 (31), 83-112

García, C., Carreón, J., Bustos, J. M., Hernández, J. y Salinas, R. (2015). Especificación de un modelo de riesgos ambientales ante el cambio climático. Entre Ciencias, 3 (6), 73 90

García, C., Carreón, J., Bustos, J. M., Hernández, J., Aguilar, J. A. y Rosas, F. J. (2014). Una aproximación sociopolítica al desabastecimiento, tarifas, subsidios y tandeos relativos a los servicios hídricos. Ensayos Pedagógicos, 11 (1), 73-98

García, C., Carreón, J., Hernández, J. y Méndez, A. (2012). Sistemas de actitudes hacia la sustentabilidad hídrica. Tecsistecalt, 4, 1-12

García, C., Carreón, J., Hernández, J., Bustos, J. M., Bautista, M., Aguilar, J. A. y Valdés, O. (2016). Social representations about tamden periurban anthropocentrism in a neighbohoord: wáter impact to of leaks in local development. Aacademia Journal of Environmental Science, 4 (6), 101-104 
Año 11.

Frontera

Núm. 27
Revista de Investigación

Académica sin

ISSN: 2007-8870

\section{http://revistainvestigacionacademicasinfrontera.com}

García, C., Carreón, J., Hernández, J., Bustos, J. M., Domínguez, G. A. y Morales, M. L. (2013). La cobertura periodística en torno a los conflictos hídricos por el desabasto de agua en una demarcación de México, Distrito Federal. Multidisciplina, 14, 21-48

García, C., Carreón, J., Hernández, J., Mejía, S., García, E. y Rosas, F. J. (2015). Hacia una agenda hídrica para la gobernanza local sustentable. Revista Internacional de Investigación en Ciencias Social, 11 (1), 130-154

García, C., Carreón, J., Hernández, J., Montero, M. E. y Bustos, J. M. (2013). Actitudes, consumo de agua y sistemas de tarifas del servicio de abastecimiento de agua potable. Polis, 12 (34), 363-401

García, C., Carreón, J., Mecalco, J., Hernández, J., Bautista, M. y Méndez, A. (2013). Estructura de las percepciones de riesgo en torno a la escasez y el desabasto de agua local y global. Xhimai, 8 (15), 95-118

García, C., Carreón, J., Mendoza, D., Aguilar, J. A., Hernández, J., Mejía, S. y Estrada, F. M. (2014). Especificación de un modelo de agenda sociopolítica en torno a los conflictos hídricos y la pacificación retributiva. Obets, 9 (2). 249-265

García, C., Juárez, M. y Bustos, J. M. (2017). La cobertura periodística en torno a los conflictos por el desabasto de agua en México, Distrito Federal. Artyhum, 41, 93-128

García, C., Juárez, M., Sandoval, F. R. y Bustos, J. M. (2017). Una aproximación psicológica a la complejidad ambiental: Especificación de un modelo de estrés y resiliencia. Comunitaria, 14, 75-95 
Año 11.

Frontera

Núm. 27
Revista de Investigación

Académica sin

ISSN: 2007-8870

\section{http://revistainvestigacionacademicasinfrontera.com}

García, C., Montero, M. E., Bustos, J. M., Carreón, J. y Hernández, J. (2013). teoría de la actitud hacia el consumo sustentable del agua. Sustentabilidades, 8, 1-12

García, C., Montero, M., Bustos, J. M. y Carreón, J. (2012). Los estilos de vida en torno a las problemáticas hídricas. Sustentabilidades, 4, 1-11

García, C., Rivera, B. L., Limón, G. A., Bustos, J. M. y Juárez, M. (2017). Especificación de un modelo de sostenibilidad consensuada. Revista Internacional de Investigación en Ciencias Sociales, 13 (2), 201-224

Sandoval, F. R., Bustos, J. M. y García, C. (2018). Contrastación exploratoria de un modelo de gobernanza de la sustentabilidad hídrica local. Gestión de las Personas y Tecnología, 31, 72-87

Vanegas, M. C., Corral, V., Bustos, J. M. y Ortega, P. (2018). Desarrollo de la escala expectativas de comportamiento ambiental de otros con adultos jóvenes mexicanos. Universitas Psychological, 17 (2), 1-10 